\title{
CRESCIMENTO INICIAL DE MUDAS DE CAFÉ CONILON NO CAMPO, PRODUZIDAS EM DIFERENTES RECIPIENTES E SUBSTRATOS ORGÂNICOS UTILIZANDO SOMBREAMENTO COM ADUBO VERDE
}

\author{
Matheus Wandermurem da Silva ${ }^{1}$ \\ Jean Karlos Barros Galote ${ }^{2}$ \\ Juliana Elias de Oliveira ${ }^{3}$ \\ Sandy Queiroz Espinoso ${ }^{4}$ \\ Diego Souza Tosta ${ }^{5}$ \\ Otacílio José Passos Rangel ${ }^{6}$ \\ Wallace Luís de Lima $^{7}$
}

\begin{abstract}
Resumo: O objetivo deste trabalho foi avaliar o desenvolvimento inicial de mudas de café conilon no campo utilizando adubação verde, produzidas em diferentes recipientes e substratos orgânicos. O experimento foi realizado no Setor de Agroecologia do Ifes Campus de Alegre, em delineamento inteiramente casualizado, em esquema fatorial duplo (2x4), sendo o tipo de recipientes (sacolinha $-650 \mathrm{~cm}^{3}$ e tubetinho $-120 \mathrm{~cm}^{3}$ ), e substratos orgânicos (Comercial; Composto de leguminosa; Composto de gramíneas e Vermicomposto). Foram produzidas 48 mudas seminais de café conilon Robusta tropical (EMCAPA 8151), cada tratamento com 6 repetições. As melhores respostas para as variáveis morfoagronômicas avaliadas, foram obtidas para as mudas oriundas de sacolinhas $\left(650 \mathrm{~cm}^{3}\right)$. A utilização de recipientes de baixo volume de substrato pode comprometer o seu desenvolvimento após transplantio para o campo e, consequentemente, aumentar o custo de produção com o replantio de novas mudas.

Palavras-chave: Cafeicultura Orgânica, Agroecologia, Leguminosas, Conservação do Solo.
\end{abstract}

\footnotetext{
${ }^{1}$ Curso Superior de Tecnologia em Cafeicultura/Instituto Federal do Espírito Santo - Ifes/Campus de Alegre, Brasil. E-mail: matheus_wandermurem@hotmail.com.

2 Pós-Graduação em Agroecologia/Instituto Federal do Espírito Santo - Ifes/Campus de Alegre, Brasil. jean-Email: karlos10@hotmail.com.

3 Engenharia Agronômica/Universidade Federal do Espírito Santo Engenharia em Agronomia, Brasil. E-mail: juliana.elias.o@hotmail.com.

${ }_{4}$ Curso Superior de Tecnologia em Cafeicultura/Instituto Federal do Espírito Santo - Ifes/Campus de Alegre, Brasil. E-mail: sandyespinoso@gmail.com.

${ }_{5}$ Curso Superior de Tecnologia em Cafeicultura/Instituto Federal do Espírito Santo - Ifes/Campus de Alegre, Brasil. E-mail: diego.s.tosta@gmail.com.

6 Professor/Instituto Federal do Espírito Santo - Ifes/Campus de Alegre, Brasil. E-mail: otaciliorangel@gmail.com.

7 Professor/Instituto Federal do Espírito Santo - Ifes/Campus de Alegre, Brasil. E-mail: limawl@yahoo.com.br.
} 